\title{
Factors Contributing to Staff's Susceptibility to HIV in Oromia Agricultural Research Institute: A Case Study from Zeway Fisheries Resources Research Center
}

\section{Tilahun Genet Abdi*}

Zeway Fisheries Resources Research Center, Zeway, Oromia Regional State, Ethiopia

\begin{abstract}
This case study was conducted during the year of 2012 on the staffs of Zeway fisheries resources research centre, Oromia. It mainly focused on identifying factors contributing to staffs susceptibility to HIV. Susceptibility refers to the likelihood of being infected by HIV. Qualitative analysis has been widely used. Data has been presented and analysed by use of the following: Discussions, tables, responses were organized together according to their types. The study identified the following as factors that contributed to susceptibility to HIV. These factors were knowledge of staffs on HIV transmission and methods of HIV prevention, decision making, and culture of silence on HIVIAIDS aspects, religious practices and beliefs, long absence from home, use of field allowance and use of stimulant drugs. The study indicated that the likelihood of staffs susceptibility to HIV with regard to their knowledge about HIV transmission and methods of HIV prevention is less likely and with regards to use of stimulant drugs, use of field allowance, long absence from home and culture of silence on HIV and AIDS aspects, the staffs were more likely to be susceptible to HIV infection. Organization need to encourage behavioural change or change from high risk to more responsible sexual behaviour. Due to having disposable field allowances and absence of better recreation centres during field work, men staffs tend to show high susceptibility by spending part of their allowances to drink alcohols. However, no way to reduce their field allowances. Therefore, organization should deal with those identified as a high susceptibility group of men to bring behavioural change. One way of dealing with this is through providing managerial level consultation.
\end{abstract}

Keywords: HIV; Susceptibility; Staffs; Zeway fisheries resources research centre; Ethiopia

\section{Introduction}

Ethiopia is low income country and its economy is largely dependent on the agriculture. The first HIV infections in Ethiopia were identified in 1984, and the first AIDS cases were reported in 1986 [1]. High prevalence of HIV/AIDS has been the most challenging problem in developing countries, especially in sub-Saharan African countries since when the epidemic is recognized in the region. Ethiopia is classified in this region, where wide spread of HIV/AIDS currently manifested and hard hit by the epidemic. Ethiopia represents a low-level, generalized epidemic driven by most at risk populations (MARPs). National projections estimate approximately 1.1 million Ethiopians are living with HIV and prevalence increased slightly to 2.3 percent by 2009 . Although the epidemic is currently stable, HIV/AIDS remains a major development challenge for Ethiopia [2].

The situation of HIV/AIDS epidemic in Oromia and some other regions (Amhara, SNNPR, and Addis Ababa) is worse than the rest of the regions in Ethiopia. Together, they accounted for $86.6 \%$ of all PLWHA (people living with HIV/AIDS), $85.3 \%$ of new infections, $87.9 \%$ of new AIDS cases, and $88.2 \%$ of AIDS deaths.

Zeway Fisheries Resources Research Centre (ZFRRC) is government organization in Oromia regional state working on fisheries and related researches. ZFRRC is one the research centres under Oromia Agricultural Research Institute. The organization had 50 regular staffs at the time of this study. The centre is found in Batu town which is located about $160 \mathrm{~km}$ south of Addis Ababa. The centre is conducting its research in the whole Oromia Regional State and hence it is the centre's mandate to work on fisheries in the region. Field staffs of the organization are working in these mandate areas by being away from their home during the months of the year. The researcher is currently working in fishery technology extension team. His main responsibilities are technology verification, promotion and training the beneficiaries of the technologies. Based on the information above, this research focuses on identifying factors contributing to staff's susceptibility to HIV infection during their work away from home and at office level.

\section{Research Design and Methodology}

The research was carried out in Zeway town during the year 2012 Zeway is a town and separate district in central Ethiopia. It is located on the road connecting Addis Ababa to Nairobi in the East Shewa Zone of the Oromia Region of Ethiopia. In this town, all the government sector bureaus, private organizations, and non-governmental organizations and other community based organizations are found. Zeway Fisheries Resources Research Centre (ZFRRC) and its staffs, where this study focused are found in this town. However, the staffs of ZFRRC most of the time were working in the whole Oromia regional state travelling to the distant working areas. Zeway fisheries resources research centre was under Oromia Agricultural Research Institute (OARI). OARI was a regional research institute and had 18 research centres. From these eighteen research centres, ZFRRC was the only centre working on fisheries in the whole Oromia regional state. This was one of the reasons why ZFRRC was selected for the purpose of this study because of their working situations and hence the staffs were widely moving for research purposes and the factors contributing to their susceptibility to

*Corresponding author: Tilahun Genet Abdi, Zeway Fisheries Resources Research Center, Ziway, Ethiopia, Tel: +251912164429; E-mail: tilagenet2006@yahoo.com

Received October 30, 2013; Accepted November 27, 2013; Published December 05, 2013

Citation: Abdi TG (2013) Factors Contributing to Staff's Susceptibility to HIV in Oromia Agricultural Research Institute: A Case Study from Zeway Fisheries Resources Research Center. J AIDS Clin Res 4: 266. doi:10.4172/2155 6113.1000266

Copyright: ( $) 2013$ Abdi TG. This is an open-access article distributed under the terms of the Creative Commons Attribution License, which permits unrestricted use, distribution, and reproduction in any medium, provided the original author and source are credited. 
HIV during their field work need to be identified. The whole staffs of the organization were also considered for the purpose of this study. This is because little was known about staffs' susceptibility to HIV infection and the organization need to know the factors that were contributing to staffs susceptibility to HIV infection at work place.

\section{Secondary data collection}

Secondary data was collected through reviewing different literatures. These literatures were obtained from internet search, books, articles, journals and proceedings of different workshops. These secondary data assisted in coming up with the concepts and guided the research activities.

\section{Primary data collection}

Thirty respondents were identified. Nineteen field staffs and 11 support staffs were identified from the same organization for the purpose of this study. All respondents from field staffs were purposively identified because they had more exposures to field works in the whole Oromia regional state. However, the other support staffs were selected randomly to get additional information about the study at work place.

NB. Support staffs referred to the staffs not frequently working on field work. These include human resource work process, finance and purchase work process and planning, monitoring, evaluation and follow up work process. Field staffs referred to staffs who were frequently working on field activities away from home.

\section{Data analysis and interpretation}

Because of the nature of the study, qualitative analysis has been widely used. Data has been presented and analysed by use of the following: Discussions, tables, responses were organized together according to their types. The analysis was also done by comparing numbers and grouping different respondents.

\section{Result and Discussion}

In this study susceptibility refers to the likelihood of being infected by HIV.

\section{Knowledge on HIV transmission}

The result indicated that all respondents (30) have replied to unprotected sexual intercourse with infected person, sharing infected needles and infected blood transfusion as the major transmission routes of HIV while mother to child transmission is the least $(11 / 30)$ replied (Table 1). The whole male respondents (21/21) responded unprotected sexual intercourse with infected person, sharing infected needles and infected blood transfusion as major transmission routes of HIV as also all female (9/9) respondents responded the same. Female respondents (4/9) responded better as compared to male respondent (7/21) on transmission ways of HIV through mother to child. Young age $(9 / 24)$ responded better than old age $(2 / 6)$.

There is no significance difference between male and female as well as young and old respondents with regard to the knowledge of HIV transmission. There is also no significance difference between field staffs and support staffs with regard to knowledge of HIV transmission. This was because all respondents were aware of the main transmission routes of HIV. In line with this study, the study conducted by Yeraswork Admassie and Mesfin Addisse [3] on staffs of different organization in Ethiopia revealed that staffs are well aware of the ways by which HIV is transmitted as well as the traditional practices that have helped its spread in Ethiopia.

However, only 11/30 respondents knew the HIV transmission from mother to child (MTCT) during pregnancy and breast feeding. This was because the majority of the respondents only knew HIV transmission from mother to child occurs only if there is blood contact between infected mother and child during birth. They were not aware of HIV transmission from mother to child during pregnancy and breast feeding.

The study conducted by Yeraswork Admassie and Mesfin Addisse [3] revealed that only $45 \%$ of the staffs knew about transmission from mother to child (MTCT). This report is in line with the findings of this study that transmission from mother to child is the least responded (37\%).

\section{Knowledge on methods of HIV prevention}

In order to know their knowledge of HIV prevention and the way they took measures to protect themselves, the respondents were also asked (with possibility for number of multiple responses) to list the ways and means by which they protect themselves from HIV infection. In this regard, they identified ways of HIV prevention that are listed in Table 2 below.

\begin{tabular}{|c|c|c|c|c|c|c|c|c|}
\hline \multirow{3}{*}{$\begin{array}{l}\text { Indicators } \\
\text { (Transmission routes of HIV) }\end{array}$} & \multicolumn{2}{|c|}{ Sex } & \multicolumn{2}{|c|}{ Age } & \multicolumn{4}{|c|}{ Staff's category } \\
\hline & \multirow{2}{*}{$M N=21$} & \multirow{2}{*}{$F \mathrm{~N}=9$} & \multirow{2}{*}{$\begin{array}{l}\text { Young (19-35) } \\
\mathrm{N}=24\end{array}$} & \multirow{2}{*}{$\begin{array}{l}\text { Old ( } 36 \text { and } \\
\text { above) } N=6\end{array}$} & \multicolumn{2}{|c|}{ FS } & \multicolumn{2}{|c|}{ ss } \\
\hline & & & & & $M N=18$ & $\mathrm{~F} \mathrm{~N}=1$ & $M N=3$ & $F \mathrm{~N}=8$ \\
\hline $\begin{array}{l}\text { Unprotected sexual intercourse with } \\
\text { infected person }\end{array}$ & 21 & 9 & 24 & 6 & 18 & 1 & 3 & 8 \\
\hline Sharing infected needles & 21 & 9 & 24 & 6 & 18 & 1 & 3 & 8 \\
\hline Infected blood transfusion & 21 & 9 & 24 & 6 & 18 & 1 & 3 & 8 \\
\hline Mother to child transmission & 7 & 4 & 9 & 2 & 6 & --- & 1 & 4 \\
\hline
\end{tabular}

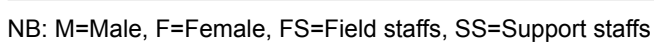

Table 1: Respondents' knowledge on transmission of HIV by sex, age and staff's category.

\begin{tabular}{|c|c|c|c|c|c|c|c|c|}
\hline \multirow{3}{*}{$\begin{array}{l}\text { Indicators } \\
\text { HIV Prevention mechanism }(\mathrm{N}=30)\end{array}$} & \multicolumn{2}{|c|}{ Sex } & \multicolumn{2}{|c|}{ Age } & \multicolumn{4}{|c|}{ Staff's category } \\
\hline & \multirow{2}{*}{$M N=21$} & \multirow{2}{*}{$\mathrm{F} \mathrm{N}=9$} & \multirow{2}{*}{$\begin{array}{c}\text { Young (19-35) } \\
\mathrm{N}=24\end{array}$} & \multirow{2}{*}{$\begin{array}{l}\text { Old ( } 36 \text { and } \\
\text { above) } N=6\end{array}$} & \multicolumn{2}{|c|}{ FS } & \multicolumn{2}{|c|}{ sS } \\
\hline & & & & & $M N=18$ & $\mathrm{~F} \mathrm{~N}=1$ & $M N=3$ & $F N=8$ \\
\hline Taking care of infected needles & 21 & 9 & 24 & 6 & 18 & 1 & 3 & 8 \\
\hline Abstinence and faithful to partner & 21 & 9 & 24 & 6 & 18 & 1 & 3 & 8 \\
\hline Use of condom & 10 & --- & 10 & --- & 9 & - & 1 & - \\
\hline Know self-status through HIV test & 1 & 3 & 3 & 1 & 1 & - & - & 3 \\
\hline
\end{tabular}

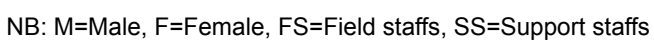

Table 2: Staffs' knowledge on prevention mechanism of HIV by sex, age and staff's category. 
There is no significance difference between male and female, young age and old age, field staffs and support staffs with regard to prevention of HIV infection through taking care of infected needles and abstinence and faithful to partner. This was because all respondents were aware of these prevention mechanisms of HIV though some of them still need more awareness about correct and consistent use of condom as a HIV prevention mechanism.

All male and females respondents from both field staffs and support staffs were aware of taking care of infected needles and abstinence and faithful to partner as a prevention mechanism of HIV infection. However, among male respondents only 10 of them knew the correct use of condom. From the rest (11/21) of the respondents, some did not know the correct use of Condom and the other did not want to talk about condoms. This may be because there was no awareness given for them on how to use condom properly. All the female staffs focused on other HIV prevention methods (taking care of infected needles and abstinence and faithful to partner) and do not want to talk about use of condom. This is might be because use of condom sometimes seen as lack of trust between sexual partners. The study conducted by Rachel [4] revealed that using or not using a condom is not simply a question of safer sexual behaviour: it is the outcome of a negotiation between partners.

The study finding showed that abstinence and faithful to partner was the most responded as prevention mechanism to HIV infection while use of condom was the least (10/30) responded from the three HIV prevention mechanisms. This was because the majority of the respondents have the awareness about prevention mechanisms of HIV infection though some of them did not know the correct use of condom. This indicated that the staffs had knowledge on the major prevention mechanism of HIV infection. HAPCO and GAMET [1] also stated that condoms, abstinence, and limiting the numbers of partners are the most frequently named methods of preventing HIV.

\section{Decision making}

This refers to the situation in the organization which consider how much the organization ensure that HIV and AIDS and gender equality issues are seriously taken into consideration in decision making on organizational matters.

In this regard, the study revealed that there were no female staffs in decision making currently in the organization. This can be a factor for the susceptibility to HIV infection. This is because Male domination affect gender responsiveness of policies and increase the gender related barriers that deny women from participating in decision making structures and processes related to issues of HIV and AIDS.

According to Maksuda [5], women's active participation in decision making is not only important for ensuring equality, but also for establishing their right addressing their problem and challenges they faced in their workplace as well as social life. Maksuda [5] further revealed that differences between men and women will also be reduced if organization exercises the participatory decision making process, develop gender policy and initiate appropriate dissemination process of policy and norms through their governance system. At the same time, the commitment of employee, especially those at the top, to gender equality and to adopting and implementing existing and future reforms is also critical for ensuring women's participation in decision making.

According to $\mathrm{WHO}[6]$ gender plays an integral role in determining and individual's susceptibility to infection, his or her ability to access care, support or treatment and ability to cope when infected or affected.

\section{Culture of silence on HIV/AIDS}

The organization had a kind of process culture. The employees were following the process and procedures of the organization. Feedback and reviews of the activities were held quarterly in the organization. In such a situation, staffs were discussing and openly sharing their views about work activities in the organization and at field work. However, the study revealed that staffs were silent to discuss about HIV/AIDS, sex and gender equality. This may be because they were not familiar with HIV and AIDS aspects as a concern besides their research activities due to insufficient awareness about it.

Report from Prem [7] revealed that some socio-cultural norms prevent both women and men from obtaining critical information about HIV/AIDS. For example, there was culture of silence around sexual matters. In line with this report, the findings from focus group discussion and respondents revealed that there was culture of silence in the organization to talk about sexual matters. However, both women and men are important players in preventing HIV and all people benefit from responsible, respectful, consensual and mutually relationships [2].

\section{Religious practices and beliefs}

The study revealed that the majority of respondents responded as religious practices or beliefs encourages in reducing the risk of HIV infection. For their justification to their response some of the Christian respondents repeatedly talked about the bible verse which supports their idea:

"You have heard that it was said to those of old 'you shall not commit adultery.' But I say to you that whoever looks at a woman to lust for her has already committed adultery with her in his heart'. Matthew 5: 27-28".

According to the respondents those who have high respect to their religious rules and principles, their intention regarding the above words of the bible was that a man should be faithful to his partner. As a result staffs susceptibility to HIV infection likely to be decreased.

The findings of this study indicated that the majority of the respondents supported religious practices or beliefs encourages in reducing HIV infection for the above stated reasons even though some of them were not stick to their religious rules.

Koenig [8] stated that religious beliefs or practices are associated with high faithfulness to partner, higher social support and greater marital satisfaction and stability. It can be argued that the deviation from this ideal can in many cases lead to HIV infection.

\section{Long absence from home}

The majority (14/19) of field staffs replied that long absence from home had a great risk of HIV infection. Young ages (11/13) responded better than old ages (3/6) and non-married (4/5) responded better than married respondents (10/14).

According to the results from the study, young ages are more influenced by long absence from home than old ages (Table 3). This is because they are more influenced by risky behaviours (e.g. taking stimulants during the night at their field work) than old ages. For the same reason non-married respondents are more influenced by being away from home than married respondents.

The following ideas (Box 1 below) were from the respondents with regard to the risk of long absence from home for the likelihood of becoming infected by HIV during field work. 


\begin{tabular}{|l|c|c|c|c|c|}
\hline \multirow{2}{*}{$\begin{array}{l}\text { Indicators } \\
\text { Long absence from home (N=19) }\end{array}$} & \multicolumn{2}{|c|}{ Sex } & \multicolumn{2}{|c|}{ Age } \\
\hline Male N=18 & Female N=1 & Young (19-35) N=13 & Old (36 and above) N=6 & Married N=14 & Not married N=5 \\
\hline No risky situations & 14 & -- & 11 & 3 \\
\hline
\end{tabular}

Table 3: Field staffs' response on susceptibility to HIV due to long absence from home.

"Being far from home will give the chance to create disagreement with partner. Sometimes when you are far from home somebody will be at your home which creates double chance to get infected by HIV". (Married field staff)

"When I went to my fieldwork it is known that I consume food from different hotels and cafeteria which is occupied by many ladies which may attract me towards them". (One of the field staff)

"Being far from home made me the chance to drink alcohol which increases the chance to have unprotected sex with others". (One of young field staffs)

"Long absence from home can expose us to have sex with bar ladies (sex desire + availability of bar ladies + no safety tool= exposure to HIV infection)". (One of the young field staffs)

Box 1: Responses of the staffs on long absence from home and risk of HIV infection.

"The more you take stimulant drugs the more you stimulate yourself to have sex with others and high chance not to use condoms" (one of male field staffs)

"The more you take stimulant the more you lose your mentality, so you are easily attracted by any opposite sex" (one of male field staffs)

"Stimulant drugs like alcohols initiate people to do what they feel internally including sexual drives" (one of male field staffs)

Box 2: Responses of the staffs on use of stimulant drugs and risk of HIV infection.

The above Box 1 explained that how far the respondents understood the risk of HIV infection during their field work away from their home. Especially the young respondents' response had an indication that being away from home for long time had a great risk for HIV infection.

The researcher's finding is more agreed with the study according to Hapco and Gamet [1], mobile workers such as civil servants, truck drivers, seasonal workers and others who spend a portion of their time away from residence become more susceptible to be contracted with HIV.

\section{Use of field allowance}

All respondents from field staffs revealed that they spend their allowances for food and rent for bed room. However, they also revealed that there was a difference of field environment where they can use their field allowance and for what purpose. In this regard, most of the respondents indicated rift valley areas of the Oromia Regional state as the place where the staffs most of the time spend part of their allowance for drinking alcohols. The majority of the respondents from young age were more enjoying during the night after their field work through drinking alcohols. The respondent themselves confirmed that the more they take stimulant alcohols the more they stimulate themselves to have sex with others and high chance not to use condoms. This can increase their susceptibility to HIV infection.

The respondent replied that 'the more you stayed in town the more you are engaged in risky situation in that there is the chance of taking stimulant alcohols which in turn initiates you to have unprotected sex that increases the likelihood of being infected by HIV'.

\section{Use of stimulant drugs}

Use of stimulant drug in this study refers to drinking alcohols and sometimes smoking shisha. In this regard, respondents were asked whether this stimulant drugs influenced their behaviour to have unprotected sex.

The above Box 2 shows the extent to which respondents recognized the effect of stimulant drugs in initiating someone to sexual desires and leads to unprotected sex.

According to Njue et al. [9] alcohol and drug use facilitated and reinforced sexual risky behaviours. The link between alcohol abuse and sexually transmitted infections (including HIV infection) has been the subject of discussion in many studies (e.g. Kalichman et al. [10] in South Africa, Mmbaga et al. [11] in Tanzania and Kalichman et al. [12]). What this study has in common is that alcohol use predisposes individuals to greater risk of HIV infection. In this study, respondents' perceptions reflect this general understanding.

Judging from informants' comments, when people are drunk, casual sex is far more likely. A few people said they did not know if they have had a one-night stand because they were too drunk to remember, let alone remember if they used a condom [13].

Therefore, stimulant drugs have high influencing power to increase sexual feelings of few staffs to have casual sex with available ladies and then to be susceptible to HIV infection if they are not using condom during sexual intercourse.

\section{Conclusion and Recommendation}

Knowledge of staffs on transmission routes of HIV and prevention mechanism of HIV was good according to the result of the study though some of the respondents have limited knowledge regarding correct use of condom. Long absence from home and influence of field work had high risk for field staffs for the likelihood of becoming infected by HIV. The more the field staffs stayed in field work the more their susceptibility to HIV increased due to the influence of being absent from home for long time and the influence of field environment. In this regard, almost all field staffs were highly susceptible to HIV for their long absence from their home even though young ages were more susceptible than others.

Organization need to encourage behavioural change or change from high risk to more responsible sexual behaviour. Due to having disposable field allowances and absence of better recreation centres during field work, men staffs tend to show high susceptibility by spending part of their allowances to drink alcohols. However, no way to reduce their field allowances. Therefore, organization should deal with those identified as a high susceptibility group of men to bring behavioural change. One way of dealing with this is through providing managerial level consultation. 
Citation: Abdi TG (2013) Factors Contributing to Staff's Susceptibility to HIV in Oromia Agricultural Research Institute: A Case Study from Zeway Fisheries Resources Research Center. J AIDS Clin Res 4: 266. doi:10.4172/2155-6113.1000266

The organization should encourage women in decision making. This will reduce differences between men and women if organization exercises participatory decision making process, develop gender policy and initiate appropriate dissemination process. This is because male domination affects gender responsiveness of policies and increase the gender related barriers that limit women from participating in decision making structures and process related to issues of HIV and AIDS. One way of doing this is by empowerment of women through training on leadership and management aspects.

\section{References}

1. HAPCO, GAMET (2008) HIVIAIDS in Ethiopia. An Epidemiological Synthesis HIVIAIDS Prevention and Control Office (HAPCO). Global AIDS Monitoring and Evaluation Team (GAMET)

2. Gender equality is a key to workplace responses to HIV and AIDS (2010) UNAIDS.

3. Yeraswork Admassie, Mesfin Addisse (2009) Managing HIV and AIDS in the work place, in Ethiopia. STOP AIDS NOW Project. Fact sheet applied research phase one.

4. Rachel M, Mash B, Pierre de Villiers (2010) 'Why don't you just use a condom?': Understanding the motivational tensions in the minds of South African women. African Journal of Primary Health Care \& Family Medicine 2.
5. Maksuda A (2010) Effect of organizational governance on women's participation in decision making. North South University, Bangladesh.

6. WHO (2003) Integrating Gender into HIVIAIDS programmes. Department of Gender and Women's Health, Family and Community Health, World Health Organization.

7. Prem (2003) HIVIAIDS and Gender Equality. Gender and Development Briefing Notes. Partnership for Research and Education in Materials.

8. Koenig HG (2004) Religion, spirituality, and medicine: research findings and implications for clinical practice. South Med J 97: 1194-1200.

9. Njue C, Voeten HA, Remes $P$ (2009) Disco funerals: a risk situation for HIV infection among youth in Kisumu, Kenya. AIDS 23: 505-509.

10. Kalichman SC, Simbayi LC, Cain D (2010) HIV transmission risk behaviours among HIV seropositive sexually transmitted infection clinic patients in Cape Town, South Africa. Eur J Public Health 20: 202-206.

11. Mmbaga EJ, Hussain A, Leyna GH, Mnyika KS, Sam NE, et al. (2007) Prevalence and risk factors for HIV-1 infection in rural Kilimanjaro region of Tanzania: implications for prevention and treatment. BMC Public Health 7: 58.

12. Kalichman SC, Simbayi LC, Kaufman M, Cain D, Jooste S (2007) Alcohol use and sexual risks for HIVIAIDS in sub-Saharan Africa: systematic review of empirical findings. Prev Sci 8: 141-151.

13. Debie LB, Yoder PS (2008) Alcohol Consumption, Sexual Partners, and HIV Transmission in Namibia. USAID, ICF Macro, Calverton, Maryland, USA. 